\title{
Histoplasmose felina disseminada: relato de um caso
}

\author{
$\underline{\text { Elissandra da Silveira }}{ }^{1}$; Iasmine Biz Mottin $^{2}$; $\underline{\text { Adrielly Ehlers }}^{3}$; Sandra Márcia Tietz \\ Marques $^{4}$ \\ ${ }^{1}$ Médica Veterinária, Residente de Clínica Médica de Pequenos Animais do Hospital de Clínicas Veterinárias, Faculdade de \\ Veterinária, Universidade Federal do Rio Grande do Sul, RS. \\ ${ }^{2}$ Médica Veterinária, Residente de Clínica Médica de Pequenos Animais do Hospital Veterinário da ULBRA, Universidade \\ Luterana do Brasil, Canoas, $R S$. \\ ${ }^{3}$ Médica Veterinária, Residente de Patologia do Hospital Veterinário da ULBRA, Universidade Luterana do Brasil, Canoas, \\ $R S$. \\ ${ }^{4}$ Médica Veterinária, Doutora em Ciências Veterinárias, Departamento de Patologia Clínica Veterinária, Faculdade de \\ Veterinária, Universidade Federal do Rio Grande do Sul, Porto Alegre, RS. \\ *Autor para correspondência: E-mail: smtmuni@hotmail.com
}

RESUMO: Descreve-se um caso de histoplasmose disseminada em um felino fêmea com dois anos de idade, que residia em um sítio no município de São Francisco de Paula, Rio Grande do Sul. O animal apresentava lesões ulceradas e nodulares nas junções mucocutâneas palpebral, oral, anal, na língua e palato duro a cerca de duas semanas. Foram executados: exame de sangue, radiografia torácica, biópsia incisional, cultura micológica e exame histopatológico. Ao exame histopatológico a lesão é constituída por reação inflamatória piogranulomatosa difusa com predomínio de macrófagos contendo numerosas formas fúngicas e caracterizada no exame micológico como Histoplasma capsulatum.

Palavras chave: gato, histoplasmose, pneumonia fúngica, testes diagnósticos.

\section{Disseminated feline histoplasmosis: a case report}

\begin{abstract}
We describe a case of disseminated histoplasmosis in a female cat with two-year-old, who lived on a farm in the municipality of San Francisco de Paula, Rio Grande do Sul. The animal had ulcerated and nodular lesions in the eyelid muco cutaneous junctions, oral, anal, tongue and hard palate about two weeks. Were performed: blood test, chest radiograph, incisional biopsy, mycological culture and histopathological examination. Histopatological injury examination consists of pyogranulomatous diffuse inflammatory reaction with predominance of macrophages containing numerous fungal forms and characterized in mycological examination as Histoplasma capsulatum.
\end{abstract}

Keywords: cat, histoplasmosis, fungal pneumonia, diagnostic tests.

\section{Introdução}

A histoplasmose é uma micose sistêmica, de caráter oportunista, causada por um fungo dimórfico, Histoplasma capsulatum que afeta seres humanos e animais ao redor do mundo (Klang et al., 2013). Duas variedades do $H$. capsulatum são conhecidas: var. capsulatum e var. duboisii. Ambas são indistinguíveis em sua forma miceliana, mas diferem na forma leveduriforme; na var. duboisii, as células são maiores e têm paredes mais espessas que na var. capsulatum (Ferreira \& Borges, 2009). Seu habitat é o solo contendo fezes de aves e morcegos, que servem como um bom meio de crescimento para o organismo, podendo persistir no ambiente, após a contaminação, por longos períodos de tempo. As aves não albergam o fungo devido à alta temperatura corporal, mas os morcegos podem ser portadores crônicos, excretando formas viáveis em suas fezes.

Histoplasma capsulatum pode ser considerado um dos patógenos oportunistas mais importantes do homem. A histoplasmose humana, também denominada de doença de Darling, é considerada zoonose pela Organização Mundial de Saúde (Carneiro et al., 2005). Pacientes com linfomas, 
transplantados renais, cardíacos e hepáticos, pacientes em uso de altas doses de corticosteróides e, particularmente, doentes com AIDS podem desenvolver quadros graves de histoplasmose disseminada, resultantes da recrudescência de infecções latentes e, menos frequentemente, de reexposição ao microrganismo em áreas hiperendêmicas (Ferreira \& Borges, 2009).

Infecção ocorre pela inalação de conídeos e pode ser subclínica, mas também causa doença pulmonar, que por via hematógena ou linfática se dissemina para diversos órgãos, com afinidade pelo sistema reticulo endotelial resultando em hepatomegalia e esplenomegalia (Klanget al., 2013). Os sinais clínicos em gatos, como fraqueza, letargia, febre, anorexia, emaciação, sinais oculares, envolvimento esquelético e sinais respiratórios são frequentemente crônicos e inespecíficos apesar da doença disseminada (Brömel \& Sykes, 2005; Carneiro et al., 2005). Os gatos infectados podem apresentar-se clinicamente sadios ou desenvolver doença disseminada. A maioria dos casos ocorre em animais com menos de quatro anos de idade (Nelson \& Couto, 2001). O diagnóstico da histoplasmose baseia-se em exames histopatológicos, sorológicos e testes alérgicos, porém o diagnóstico definitivo é feito mediante cultura (Carneiro et al., 2005). O objetivo deste relato é descrever um caso de histoplasmose sistêmica em gato.

\section{Relato de um caso}

Uma gata com dois anos de idade, de raça mista, castrada, vacinada contra rinotraqueíte, calicivírus e panleucopenia foi atendida em um Hospital Veterinário da região metropolitana de Porto Alegre, Rio Grande do Sul, Brasil, em 2015. O felino residia em um sítio no município de São Francisco de Paula. A razão da consulta eram as lesões nas pálpebras, boca e ânus há duas semanas. O tutor do animal relatou que já havia consultado outro veterinário, que prescreveu doxiciclina (5mg/kg a cada 12 horas), mas as lesões continuavam progredindo.

Ao exame clínico, a gata apresentava-se hidratada, normotérmia, frequência cardíaca e respiratória dentro do padrão para a espécie, palpação abdominal sem alteração e mucosas normocoradas. Evidenciavam-se lesões ulceradas e nodulares nas junções mucocutâneas (palpebral, oral e anal), na língua e palato duro (Figural A, B e C). Com suspeita clínica de histoplasmose, foram requeridos hemograma, bioquímica renal e hepática, radiografia torácica e biópsia incisional das lesões palpebral, oral e anal, sob sedação com meperidina $(3 \mathrm{mg} / \mathrm{kg})$, cetamina $(6 \mathrm{mg} / \mathrm{kg})$, midazolan $(0,3 \mathrm{mg} / \mathrm{kg})$ e propofol $(3 \mathrm{mg} / \mathrm{kg})$, por via endovenosa.
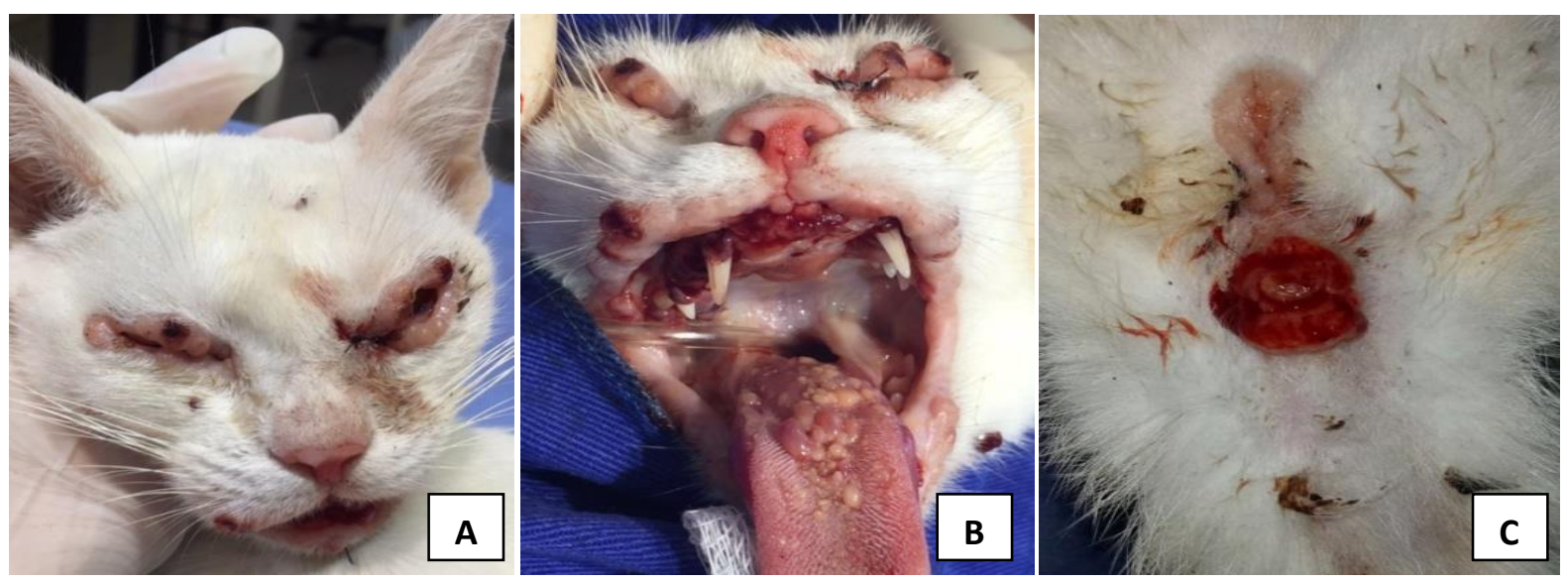

Figura 1 A: lesões ulceradas e nodulares nas junções mucocutâneas palpebral e perioral; B: Lesões em base e palato duro; C: lesão perianal.

\section{Resultados e discussão}

O exame sanguíneo apresentou uma anemia grave e por esse motivo, a paciente realizou transfusão sanguínea. O eritrograma mostrou redução em: eritrócitos $\left(3,0 \mathrm{x} 10^{6} / \mu \mathrm{L}\right)$; hemoglobina $(3,9 \mathrm{~g} / \mathrm{dL}$; hematócrito $(18 \%)$ e
CHCM (21,7 g/dL); e aumento em: VCR $(58,4$ fL) e RDW (19,8\%). O leucograma apresentou-se dentro dos parâmetros de normalidade bem como o exame bioquímico (creatinina, uréia e fosfatase alcalina). $\mathrm{O}$ laudo do raio $\mathrm{X}$ evidenciou pneumonia fúngica e a cultura micológica da 
mucosa oral e da lesão proliferativa no ânus evidenciou elementos leveduriformes, caracterizado como macroconídeo tuberculado maduro de Histoplasma capsulatum (Figura 2 A).

Infecção com $H$. capsulatum usualmente ocorrem pela inalação de microconidia pelo hospedeiro, depositando nos alvéolos e rapidamente se convertendo na forma de levedura nos tecidos. Esta germinação e conversão podem ocorrer previamente ou após a ingestão por macrófagos pulmonares. Uma vez dentro dos macrófagos, se multiplicam e vão para os linfonodos hilar ou mediastínicos, ganhando acesso a circulação sanguínea para disseminar-se por vários órgãos (Guimarães et al., 2006), podendo envolver fígado, baço e medula óssea. Um estudo retrospectivo com imagens de ultrassom de baço de 15 gatos com histoplasmose documentou esplenomegalia em todos os animais e aparência hipoecóica em 94\% dos casos (Atiee, 2014).

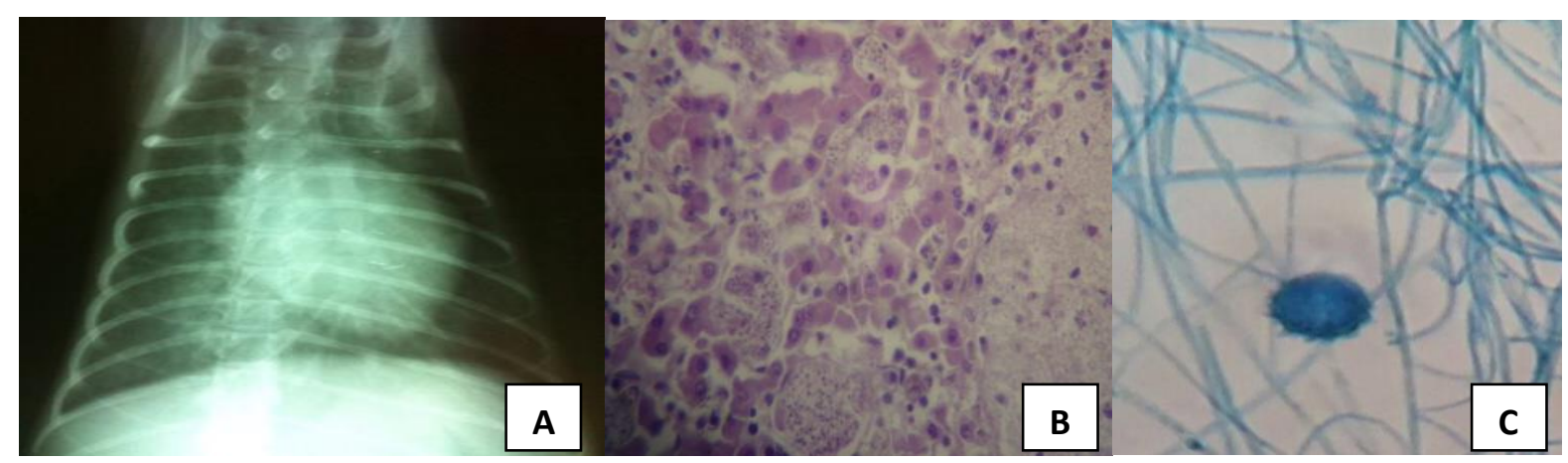

Figura 2 A: Radiografia de tórax evidenciando padrão pulmonar alveolar; B:Histologia do fígado, apresentando inflamação piogranulomatosa com macrófagos e microrganismos compatíveis com Histoplasma capsulatum, coloração HE, aum. 400 x; C:Micromorfologia do isolado Histoplasma capsulatum. Colônias isoladas em ágar $\mathrm{BHI}$, conidiogênese de micélio a $25^{\circ} \mathrm{C}$.

A biópsia evidenciou lesão constituída por reação inflamatória piogranulomatosa difusa com predomínio de macrófagos contendo numerosas formas fúngicas semelhantes à Histoplasma capsulatum (Figura 2B). O exame micológico foi confirmatório e o laudo evidenciou cultivo de Histoplasma capsulatum (Figura 2C).

Neste momento a paciente começou a ser tratada com itraconazol $5 \mathrm{mg} / \mathrm{kg}$ a cada 12 horas, visto que o tutor não autorizou a eutanásia, mesmo sendo alertado que se tratava de uma zoonose. Uma semana após o início do tratamento com o antifúngico, a paciente começou a se mostrar apática e com hipofagia. $\mathrm{O}$ tutor procurou imediatamente um serviço veterinário, porém a paciente veio a óbito. A paciente passou por necropsia, tendo como achados macroscópicas lesões proliferativas, ulceradas, evidenciando áreas necróticas na região de pálpebras, gengiva, lábios e língua. $\mathrm{Na}$ cavidade torácica foi observado pulmão com consistência firme e lesões nodulares difusas (Figura 3A). Na cavidade abdominal foi observado hepatomegalia, aumento do padrão lobular e lesões nodulares difusas (Figura 3 B) e baço com lesões semelhantes.

No quadro sistêmico, os animais podem apresentar anemia normocítica e normocrômica não regenerativa, devido à infecção medular pelo parasito, causando diminuição da função medular normal, processo inflamatório crônico suprimindo a eritropoiese e hemorragias gastrintestinais. Pode ocorrer trombocitopenia devido à coagulação intravascular disseminada (CID) ou destruição microangiopática e pancitopenia por afecção medular. Alterações bioquímicas como azotemia, hipoproteinemia, aumento de fosfatase alcalina, hiperbilirrubinemia e hiperglobulinemia podem ser observadas. Os gatos infectados podem apresentar-se clinicamente sadios ou desenvolver doença disseminada (Nelson \& Couto, 2001).

Vários animais, domésticos e silvestres, podem ser hospedeiros do Histoplasma capsulatum. No Brasil o primeiro caso foi relatado em 1946 em um cão no estado do Pará. Desde então, são poucos casos registrados (32) em cães e gatos, nas regiões sudeste, centro-oeste e nordeste, infectando apenas cães e gatos; 
entretanto a literatura descreve casos em equídeos, roedores, marsupiais, gazelas e aves (Teles et al., 2014).

Conforme Souza et al. (2015) somente quatro casos dessa doença foram relatados em gatos domésticos no Brasil; este relato é o quinto caso de histoplasmose com envolvimento supra orbital em um gato domiciliado, sem acesso à rua, porém recolhido das ruas havia três anos. Enquanto os relatos no Brasil são raros, a histoplasmose felina é a segunda doença micótica mais comum em gatos nos Estados Unidos, principalmente em gatos jovens (Atiee al., 2014). Geralmente, as regiões de maior endemicidade são as de clima moderado com umidade constante (Guimarães et al., 2006).

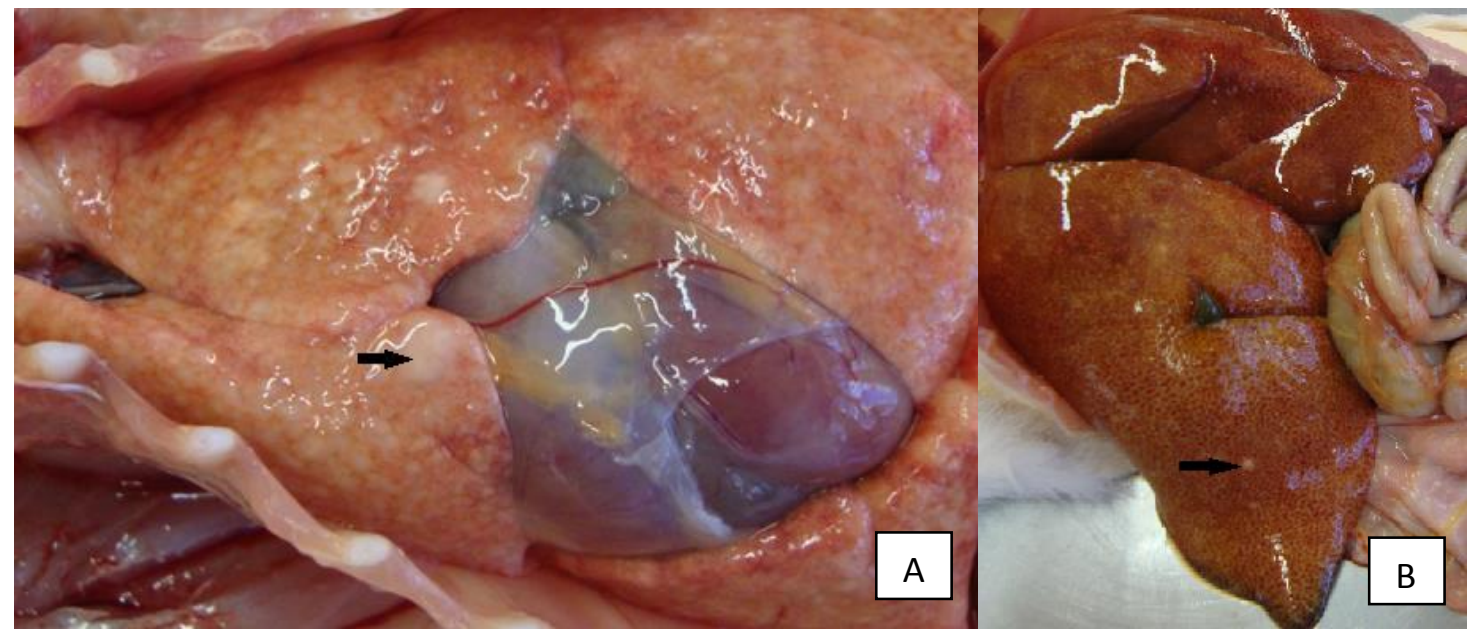

Figura 3 A: Pulmão de coloração pálida com lesões nodulares difusas (seta); B: Hepatomegalia, aumento do padrão lobular e lesões nodulares difusas (seta).

A identificação de animais domésticos e silvestres naturalmente infectados por $H$. capsulatum é uma ferramenta importante para monitorar a ocorrência do fungo em uma determinada região, pois estes atuam como sentinelas (marcadores epidemiológicos) para a presença do microrganmo, indicando a existência de fontes de infecção para humanos e animais (Emmons, 1950; Teles et al., 2014).

Conflito de interesse: os autores declaram não haver nenhum conflito de interesse.

\section{Referências bibliográficas}

Atiee, G.,Kvitko-White, H., Spalding, K. \& Johnson, M. (2014). Ultrasonographic appearance of histoplasmosis identified in the spleen in 15 cats. Veterinary Radiology Ultrasound, 55, 310-314.

Brömel, C. \& Sykes, J. E. (2005). Histoplasmosis in dogs an dcats. ClinicalTechniquesinSmall AnimalPractice, 20, 227-232.

Carneiro, R. A., Lavalle, G. E. \& Araújo, R. B. (2005). Histoplasmose cutânea em gato: relato de caso. Arquivo Brasileiro de Medicina Veterinária e Zootecnia, 57, 158-161.
Emmons, C. W. (1950). Histoplasmosis: Animal reservoirs and other sources in nature of the pathogenic fungus, Histoplasma. American Journal of Public Health, 40, 436-440.

Ferreira, M. S. \& Borges, A. S. (2009). Histoplasmose. Revista da Sociedade Brasileira de Medicina Tropical, 2, 192-198.

Guimarães, A. J., Nosanchuk, J. D. \& Zancopé-Oliveira, R. M. (2006). Diagnosis of histoplasmosis. Brazilian Journal of Microbiology, 37, 1-13.

Klang, A., Loncaric, I., Spergser, J., Eigelsreiter, S. \& Weissenböck, H. (2013). Disseminated histoplasmosis in a domestic cat imported from the USA to Austria. Medical Mycology Case Reports, 2, 108-112.

Nelson, R. W. \& Couto, C. G. (2001). Medicina interna de pequenos animais. 2. ed. Rio de Janeiro: Guanabara Koogan. 1084p.

Souza, E. W., Gremião, I. D. F., Pereira, S. A., Cunha, S. C. S. \& Borba, C. M. 
(2015). Felinehistoplasmosis: a case of Recebido em Novembro 27, 2015 $\begin{array}{lll}\text { supraorbital involvement. Revista } & \text { Brasileira de Ciência Veterinária, } & 22,77-\end{array}$ 80.

Teles, A. J., Gomes, A. R., Cabana, A. L. et al. Aceito em Janeiro 6, 2016

License information: This is an open-access article distributed under the terms of the Creative Commons Attribution License, which permits unrestricted use, distribution, and reproduction in any (2014). Histoplasmose em cães e gatos no medium, provided the original work is properly cited. Brasil. Science and Animal Health, 2, 50-66. 